\title{
PROTEOMIC PROFILE OF PULMONARY AND EXTRA PULMONARY TB SAMPLES
}

\author{
Kumar J',Cheekavolu C ${ }^{2}$,Ashalatha $\mathrm{VL}^{3}$, Leela $\mathrm{P}^{4}$, Daginawala $\mathrm{HF}^{5}$ \\ ${ }^{1}$ Lecturer, Department of Biochemistry, Maitri Dental College and Research Centre, Durg, Chhattisgarh, \\ India \\ ${ }^{2}$ Assistant Professor, Department of Pharmacology, Kerala Medical College and hospital, Mangode, \\ Palakkad, Kerala, India \\ ${ }^{3}$ Assistant Professor, Department of Biochemistry, Kerala Medical College and hospital, Mangode, \\ Palakkad, Kerala, India \\ ${ }^{4}$ PG Scholar, Department of Biochemistry, SVIMS, Tirupati, Andhra Pradesh, India \\ ${ }^{5}$ Scientist and Senior Consultant, Biochemistry Research Laboratory,Central India Institute of Medical \\ sciences, Nagpur, Maharashtra, India
}

\begin{abstract}
Introduction: Tuberculosis (TB) is a major health problem in India, so early diagnosis and treatment of Mycobacterium tuberculosis (M.tb.) infection can prevent deaths from this pathogen. The secretion of proteins by M.tb. is important in diagnostic purposes for generation of therapeutic drugs and vaccines candidates for TB. The objective of this study was to identify the protein expression (biomarkers) in TB and Tuberculosis meningitis (TBM) using proteomic approach.

Methods: In this study, using Sodium dodecyl sulfate polyacrylamide gel electrophoresis (SDSPAGE), we analyzed the secretory proteins of M.tb. in serum, cerebrospinal fluid (CSF) samples. The identified proteins were determined by Total Lab- 100 Quantity One densitometry software.

Results: Our study showed that protein bands expressed in CSF samples reveals the presence of $72 \mathrm{kD}, 70 \mathrm{kD}, 44 \mathrm{kD}, 40 \mathrm{kD} \& 16 \mathrm{kD}$ predominantly in TBM patients compared to healthy individuals. The electrophoretogram identified $97 \mathrm{kD}, 72 \mathrm{kD}, 44 \mathrm{kD}, 38 \mathrm{kD}, 29 \mathrm{kD} \& 16 \mathrm{kD}$ predominant proteins in serum samples of TB patients.

Conclusion: The detection of secretory proteins in serum and CSF samples of M.tb. in TB and TBM patients gives reliable and early diagnosis of TB and TBM. The secretory proteins can be useful as immunodiagnostic and vaccine targets which can serve as important biomarkers
\end{abstract}

Key words: Tuberculosis, Mycobacterium tuberculosis, Tuberculosis meningitis, Electrophoretogram, Biomarkers.

\section{INTRODUCTION}

Tuberculosis (TB) is one of the most challenging infectious diseases which is caused byz single

\author{
Correspondence: \\ Dr. V.L. Asha Latha \\ Assistant Professor \\ Department of Boichemistry \\ Kerala Medical College and Hospital \\ Mangode, Palakkad-679 503 \\ Kerala, India \\ E-mail: vlashalatha@gmail.com \\ Phone: +91 7025680128, 7702715179
}

infectious disease agent i.e. Mycobacterium tuberculosis (M.tb). Globally, 9.2 million new cases and 1.7 million deaths occur due to this disease which is an impediment to human development in developing countries. [1] Furthermore, due to reactivation of dormant bacteria and emergence of multi-drug resistant (MDR) strains leads to serious threat in controlling the global TB efforts. ${ }^{[2]}$ The World Health Organization (WHO) estimated the new MDR-TB cases and $60 \%$ of these cases were located in India and China. ${ }^{[3]}$ So, it is prerequisite to study the genetics and physiology of M.tb. to understand the M.tb-host interaction, to learn how 
these bacteria circumvent host defense and causes disease in order to develop new anti-tubercular agents which made a significant progress in this field. ${ }^{[4]}$

TB is a communicable disease and patients with pulmonary TB are the most important source of infection. According to the site of infections, TB can be classified as pulmonary and extrapulmonary disease. The primary route of infection involves the lungs called as pulmonary $T B$, whereas the infection causing the meninges of brain called as extra pulmonary TB. The infection of TB is initiated by inhalation of droplet nuclei $(1-5 \mu \mathrm{m})$, expectorated by patients with active pulmonary TB (open TB), typically when the patient coughs, sneeze or talking. The risk of infection is dependent on several factors such as infectiousness of the source, the closeness of contact, the bacillary load inhaled, and the immune status of potential host. ${ }^{[5]}$ Some times, the bacteria escapes from treatment of anti-TB drugs and cross blood brain barrier causes infections to meninges of host brain called tuberculosis meningitis (TBM). ${ }^{[6]}$

Early diagnosis of TB is helpful in treatment of patients and also aids in curbing the further transmission of disease. The various techniques available for the diagnosis of tuberculosis infection are microscopy (or) culture methods, fluorescent staming, antigen detection, ELISA based assays against various antigen preparation ${ }^{[7]}$,but they possess certain limitations i.e. interpretation is difficult because sensitization with nontuberculosis mycobacteria leads to false-positive tests and laborious processes etc. ${ }^{[8]}$ The set of proteins encoded by genome referred as proteomics has become a useful tool in the study of microbial physiology. This technique has been used to assess differences in levels of protein between different mycobacterial species, clinical isolates and in response to certain stimuli. ${ }^{[9]}$ Secretory proteins are the proteins released by $M . t b$. to the surrounding medium and are extremely important for diagnostic purposes and generation of therapeutic drugs and vaccines which are the useful biomarkers for immunodiagnostics of infection. ${ }^{[10,11,12]}$ It was reported that enzyme based immunoassay technique have been developed for diagnosis of pulmonary and extra pulmonary TB and promising results have been observed in these studies using crude mycobacterium extracts.
[13, 14] But, identification of mycobacterial proteins and its isolates by one and two-dimensional (2D) electrophoresis has been largely applied to broth grown cultures, because abundant protein amount are available here for both analysis and comparison. ${ }^{[15,16]}$

To the best of our knowledge the proteomic profiles of TB, TBM and its samples have been fragmentary. Thus, the present study was undertaken to observe variable pattern of proteomic profile in serum and cerebrospinal fluid (CSF) samples of M.tb. for the identification ${ }^{[3]}$ and characterization of secretory proteins as potential biomarkers.

\section{MATERIALS \& METHODS}

The study was conducted at the Research Laboratory in Central India Institute of Medical Sciences (CIIMS), Nagpur, Maharashtra, India and approved by the Institutional ethical committee and written informed consent was obtained from the patients. We prospectively selected serum samples from 20 active TB patients (13 male, 7 female; 23 61 years) and as well as 20 healthy individuals with no signs of clinical impairment and normal chest radiographs, were included as controls.

To diagnose active TB, sputum microscopy was done on two serial sputum samples by staining with Ziehl Neelson Stain as per the guidelines of India's Revised National Tuberculosis Control Programme. TB was confirmed if acid fact bacilli (AFB) and/or culture of sputum specimens were positive for M.tb. When both tests were negative, the patients were diagnosed by clinical symptoms. Clinical suspicion of tuberculosis was based on symptoms a) Chronic cough with or without expectoration or past history of TB b) Fever more than 2-3 weeks c) Progressive weight loss d) loss of appetite e) night sweats. The TBM was diagnosed based on the clinical features, which included subacute or chronic fever and signs of meningeal irritation with or without other features of central nervous system (CNS) abnormality.

\section{Specimens}

Venous blood was collected from all the patients and control subjects and allowed to clot, and after centrifugation $(1000 \times \mathrm{g}, 10 \mathrm{~min})$ the serum was separated and stored at $-20^{\circ} \mathrm{C}$ untill analysis. 
The CSF samples were confirmed on the basis of BacT/Alert-3D culturing and examination includes total and differential cell count, biochemistry, and microscopic evaluation after Gram, India ink, and AFB staining.

\section{Sodium dodecyl sulfate polyacrylamide gel electrophoresis (SDS-PAGE)}

\section{One and two dimensional electrophoresis:}

The serum and CSF samples obtained from confirmed TB and TBM patients were subjected to SDS-PAGE. SDS-PAGE was performed with a vertical slab gel electrophoresis system (Broviga, India) using the standard Laemmali method. [17] Electrophoresis was performed with vertical slab gel electrophoresis system by using $10 \%$ running gel and $5 \%$ stacking gel. The M.tb. samples are prepared by mixing with $10 \mu \mathrm{L}$ of distilled water and SDS sample buffer and incubate on heating chamber at $65^{\circ} \mathrm{C}$ for $15 \mathrm{~min}$ and electrophoresis was carried out at 250 volts $/ 50$ mAmps. After completion of electrophoresis, gels were developed using both with Coomassie brilliant blue GR-250 and silver staining method. The protein migration was allowed to proceed until the dye had migrated to the bottom of gel. The protein patterns were visualized using Total Lab-100 Quantity One densitometry software. Band size (i.e., molecular weight) was estimated using molecular weight markers (Genei, Bangalore, India) in a parallel lane.

The samples were subjected to 2DPAGE and were applied to isoelectric focusing strips with linear $\mathrm{pH}$ 4 to 7 gradients and focusing conducted using a ZOOM IPGRunner (Invitrogen, Carlsbad, CA). Strips were resolved by SDS-PAGE using 5 to $12 \%$ Bis-Tris gels (Invitrogen) followed by either staining with Coomassie or transfer to nitrocellulose. The developed gels were analyzed by using PD Quest Advanced software (Bio-Rad, Hercules, CA, USA). ${ }^{[5]}$

\section{Data analysis}

The sensitivities and specificities of serum and CSF samples for TB and TBM group were calculated. All the values obtained were expressed as mean and standard deviation (SD) and $P$-value $<0.05$ was considered as statistically significant. Statistical analysis was carried out using SPSS version 11.5 (SPSS Inc., Chicago, IL, USA) (Data not shown).

\section{RESULTS}

The present study focused on the proteins expressed by $M$. tb. in serum and CSF samples. The expressed protein profiles were studied by 1D and 2D PAGE. According to the experimental plan, we selected five serum and CSF samples of TB and TBM patients, five samples of healthy individuals and two samples as controls. The serum samples were separated out and analyzed through SDS-PAG Electrophoresis. In the gel, 13 lanes were prepared, five were serum samples of TB patients, five samples of healthy individuals and two as controls in order to compare between TB patients, healthy individuals and control samples. Gels were developed by staining with Coomassie brilliant Blue and silver staining. The gel pattern of serum fraction was then compared with the pattern of direct serum of TB patients.

The expression of low molecular weight (m.w) protein band were indicated by blue arrow whereas, red arrows shows expression of high m.w. protein. In other words, the presence of low m.w. protein in the range of approx. $29 \mathrm{kD}$ was obtained only in the serum sample of healthy individuals and absent in the serum of TB patients and control group. (Figure 1A). However, when we compare the proteomic profile of serum samples by using Quantity one densitometric software we found predominant band at $29 \mathrm{kD}$ region in TB patients whereas other samples shows the presence of predominant protein band in the region of $97 \mathrm{kD}, 72 \mathrm{kD}, 44 \mathrm{kD}$, $38 \mathrm{kD}$ as compared to healthy group(Figure 1B).

Similarly, the CSF samples of TBM patients, healthy individuals and controls were evaluated and found the presence of predominant protein bands at the region of $30 \mathrm{kD}, 68 \mathrm{kD}$ and approx. 97.4kD. In Figure-2A arrow (Green, Red, Blue, purple) indicates the expression of predominant proteins only in TBM patients as compared to healthy individuals and controls. 
Figure (1) One dimensional SDS-PAGE of M.tb. Serum Positive Sample \& Negative sample

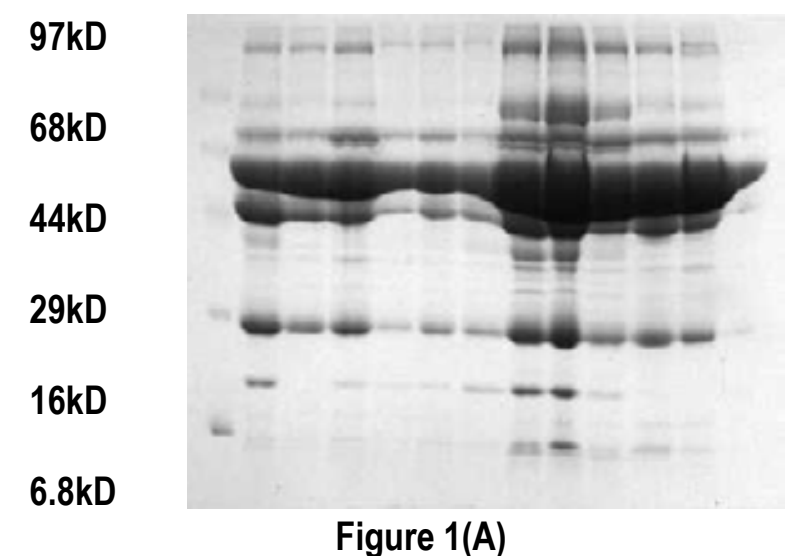

Figure 1(A)

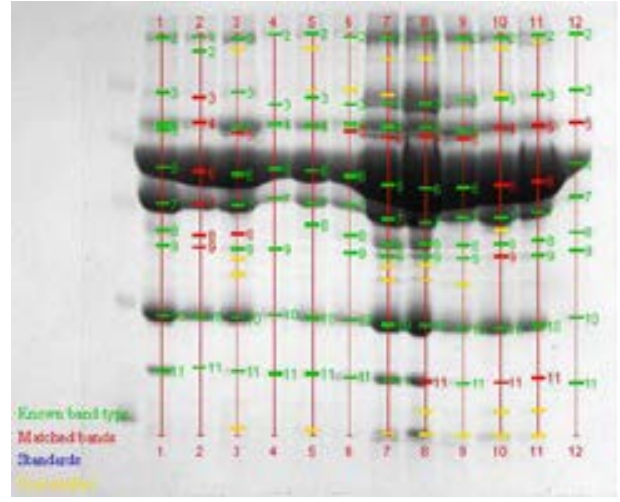

Figure 1(B)
Figure 1 A): Shows 1D SDS PAGE in M.tb. given serum positive, serum negative and the control for compare the sample.

Figure $1 \mathrm{~B}$ ): Shows densitometry analysis in 1D SDS PAGE in M.tb. given serum positive, serum negative sample and the control for compare the sample.
Lane1- Molecular wt marker

Lane2-TB 1

Lane3-TB 2

Lane4-TB 3

Lane5-TB 4

Lane6-TB 5

Lane7-NTB 1

Lane8-NTB 2

Lane9-NTB 3

Lane10-NTB 4

Lane11-NTB 5

Lane12-Control Sample

Lane13--Control Sample

Figure (2): One dimensional SDS-PAGE of CSF Positive \& CSF Negative sample 12345671234567

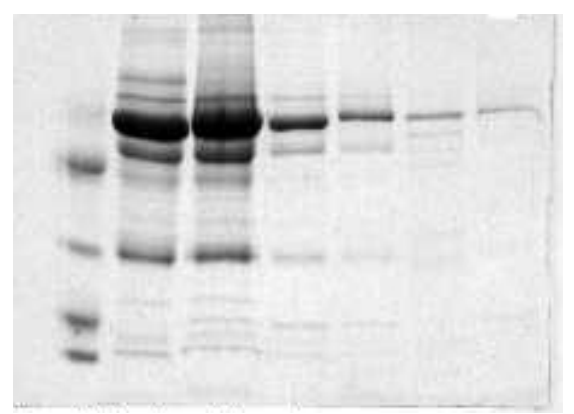

Figure 2(A)

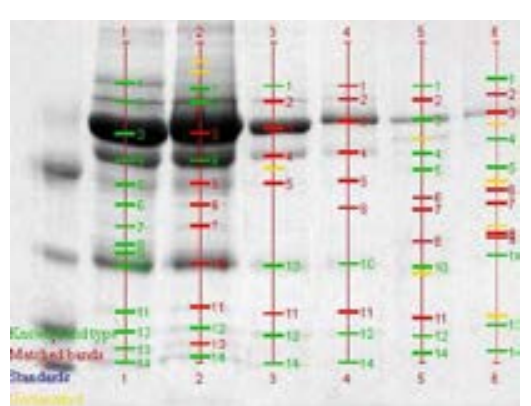

Figure 2(B)
2 A): Shows 1D SDS PAGE electrophoretogram in given CSF positive \& CSF negative and their respective healthy control sample.
2 B): Shows densitometry analysis in 1D SDS PAGE electrophoretogram in given CSF positive \& CSF negative and their respective healthy control sample.

Lane 1 - Molecular weight Marker

Lane $2 \&$ Lane 3 - TBM

Lane 4 \& Lane 5 - Non TBM

Lane 6 \& Lane 7 - CSF Control Sample 


\section{DISCUSSION}

Tuberculosis is a major health problem and reemerging as a major threat, which is rated as second most common infectious disease with high mortality rate than other infectious diseases. The diagnosis is based on relevant feature and compatible biochemical and cytological data from serum and CSF samples which varies from patient to patient. Similarly, there are various gold standard techniques available for the diagnosis of tuberculosis infections but they possess limitations like cost effectiveness, time consuming, laborious, less sensitivity and specificity.

So, as an alternative Anderson et al ${ }^{[18]}$ have suggested the use of secretory antigens as potential biomarker for serodiagnosis of tuberculosis infection. Secretory antigens are secreted by M.tb. to the surrounding medium and most potent in generation of immune response and subsequent protection against tuberculosis infection. ${ }^{[15]}$ Many researchers have attempted the use of various M.tb. antigen markers such as Lipoarabinomannan (LAM), 38kD antigen, purified protein derivatives, Antigen 85 complex, heat shock protein of $65 \mathrm{kD}$ and $14 \mathrm{kD}$ etc., which have now been recognized as the potential markers for the diagnosis of $\mathrm{TB}$

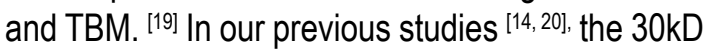
protein band identified in the CSF of TBM pateints including components of the Ag85 complex suggests that this protein can be used as important molecular marker for the early and confirmatory diagnosis of TBM.

In the present study, we detected various protein markers in the serum and CSF of patients with active TB and TBM. We evaluated and compare the CSF samples of TBM, healthy individuals and controls and found there is presence of protein bands at the region of $30 \mathrm{kD}$ and $97 \mathrm{kD}$ and also a predominant protein band below and above the albumin region (68kD) (Figure 2A \&B). In case of serum TB samples, the protein expression bands were decreased however increased in healthy individuals. As M.tb. secretes variety of protein in serum and CSF samples during their growth, these secreted proteins can be separate out through SDSPAGE electrophoresis which shows different protein pattern in different ranges. When we analyzed the serum and CSF samples with molecular weight (M.W) marker, we can easily detect the ranges of different secretory proteins with molecular weights.

In other words, the presence of low m.w. protein i.e. $29 \mathrm{kD}$ was found healthy individuals and absent in the serum of TB patients and control group. However, when we compare the proteomic profile of serum samples by using Quantity one densitometric software we found predominant band at $29 \mathrm{kD}$ region in TB patients whereas other samples shows the presence of predominant protein band in the region of $97 \mathrm{kD}, 72 \mathrm{kD}, 44 \mathrm{kD}$, $38 \mathrm{kD}$ as compared to control group (Figure $1 \mathrm{~A} \& \mathrm{~B}$ ). Our findings are in agreement with Johnson et al ${ }^{[21]}$ and Upadhye et al. ${ }^{[2]}$

We have performed electrophoretogram analysis of all serum, CSF samples of TB and TBM healthy individuals and controls which found expression of some extra protein bands, in the serum and CSF of TB or TBM patients. In short we can say that in all cases of TB, the secretory proteins of $M$. th. serve as an important diagnostic marker. These secretory proteins released during the growth of M.tb. in diseased person could be of important while differentiating from healthy individual.

Based on our study, it is clear that microorganisms secrete protein in diseased person as well as in healthy individual. The study of these secretory proteins is an important diagnostic criterion for the detection of Tuberculosis. All over the world as well in India many diagnostic methods and various techniques such as Polymerase Chain Reaction (PCR), ELISA, Western blotting etc. has been developed specifically for the diagnosis of TB and TBM. However, in our study we have performed SDS-PAGE, which was significantly helpful to compare serum and CSF samples of diseased and healthy individuals. On the basis of electrophoretogram we can conclude that in serum samples of TB patients $97 \mathrm{kD}, 72 \mathrm{kD} \& 29 \mathrm{kD}$ proteins were predominantly secreted as compared to healthy individuals. Similarly, electrophoretogram of CSF samples reveals the presence of $72 \mathrm{kD}$, $70 \mathrm{kD}, 44 \mathrm{kD}, 40 \mathrm{kD} \& 16 \mathrm{kD}$ predominantly in case of TBM patients than healthy individuals.

In conclusion, our study highlights the expression of protein bands present in serum and CSF sample of TB or TBM patients gives reliable diagnosis and does not give false results with other non- 
tuberculosis diseases. The identified protein bands are more important and useful for the diagnosis of tuberculosis which may prove as potential biomarkers in future.

\section{LIMITATION OF THE STUDY}

The characterization of identified protein bands has not been done in the present study and also the densitometric analysis of 2-D gel is not done.

\section{CONFLICT OF INTEREST}

None

\section{FUNDING}

None

\section{ACKNOWLEDGEMENT}

The authors are grateful to Dr.H.F. Daginawala, Central India Institute of Medical sciences, Biochemistry Research Laboratory, Nagpur -440010 , Maharashtra, India.

\section{REFERENCES}

1. World Health Organization: Global tuberculosis report; 2012, Available from: http://www.who.int/ tb/publications/global_report/2012/pdfffull_report. pdf.

2. Gandhi NR, Nunn P, Dheda K, Schaaf HS, Zignol $M$, vanSoolingen $D$ et al. Multidrug-resistant and extensively drug-resistant tuberculosis: a threat to global control of tuberculosis.Lancet 2010; 375:1830-1843.

3. WHO: Anti-tuberculosis drug resistance in the world. Third global report. The WHO/IUATLD Global Project on Anti-Tuberculosis Drug Resistance Surveillance (WHO/CDC/TB/2004). Geneva: World Health Organization; 2004.

4. Zhang $Y$, Telenti A. Genetics of drug resistance in Mycobacterium tuberculosis. In: Hatfull GF, Jacobs WR, editor. Molecular Genetics of Mycobacteria. Washington DC:ASM Press; 2000 .p. 235-254.

5. Frieden TR, Sterling TR, Munsiff SS, Watt CJ, Dye C. Tuberculosis. Lancet 2003; $362: 887-899$.

6. Djoba Siawaya JF, Chegou NN, van den Heuvel $\mathrm{MM}$, Diacon $\mathrm{AH}$, Beyers $\mathrm{N}$,van Helden $\mathrm{P}$ et al. Differential cytokine/chemokines and KL-6 profiles in patients with different forms of tuberculosis. Cytokine 2009; 47:132-136.
7. Nyendak MR, Lewinsohn DA, Lewinsohn DM. New diagnostic methods for tuberculosis. Curr Opin Infect Dis. 2009;22:174-182

8. Garg SK, Tiwari RP, Tiwari D, Singh R, Malhotra

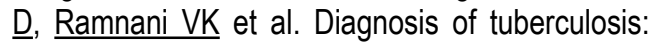
available technologies, limitations, and possibilities. J Clin Lab Anal. 2003;17:155-163.

9. Gomez M, Johnson S, Gennaro ML. Identification of secreted proteins by Mycobacteruim tuberculosis by a bioinformatic approach. Infect Immun 2000; 68: 2323-2327.

10. Starck J, Kallenius G, Marklund $\mathrm{BI}$, Andersson $\mathrm{DI}$, Akerlund T. Comparative proteome analysis of Mycobacterium tuberculosis grown under aerobic and anaerobic conditions. Microbiology 2004;150:3821-3829.

11. Bahk YY, Kim SA, Kim JS, Euh HJ, Bai GH, Cho $\mathrm{SN}$ et al. Antigens secreted from Mycobacterium tuberculosis: Identification by proteomics approach and test for diagnostic marker. Proteomics 2004; 4:3299-3307.

12. Abebe F, Holm-Hansen C, Wiker HG, Bjune G. Progress in serodiagnosis of Mycobacteruim tuberculosis Infection. Scand J Immunol 2007; 66:176-191.

13. Mehaffy $C$, Hess A, Prenni JE, Mathema B, Kreiswirth B, Dobos KM. Descriptive proteomic analysis shows protein variability between closely related clinical isolates of Mycobacterium tuberculosis. Proteomics 2010; 10:1966-1984.

14. Kashyap RS, Saha SM, Nagdev KJ, Kelkar SS, Purohit HJ, Taori GM, Daginawala HF. Diagnostic markers for tuberculosis ascites: a preliminary study. Biomark Insights 2010;5:8794.

15. Bisht $D$, Singhal $N$, Sharma $P$, Venkatesan $\mathrm{K}$. Analysis of mycobacterial strains by twodimensional gel electrophoresis. J Commun Dis 2006; 38:2552-2562.

16. Singhal N, Sharma P, Kumar M, Beenu J, Bisht D. Analysis of intracellular expressed proteins of mycobacterium tuberculosis clinical isolates. Proteome Science 2012;10:14.

17. Laemmali UK. Cleavage of structural proteins during the assembly of the head of bacteriophage T4. Nature 1970; 227:680-685.

18. Andersen $P$, Askgaard $D$, Ljungqvist $L$, Bennedsen $J$, Heron I. Proteins released from Mycobacterium tuberculosis during growth. Infect Immun 1991; 59:1905-1910.

19. Espinosa OR, Moreno JR, Jimenez AA, Maldonado RP, Paredes PA, Lopez JT. Secretion Antigens of Mycobacterium tuberculosis: A Comparison Between a Reference Strain and Seven Wild 
Isolates. Archives of Medical Research 1999; 30: 171-178.

20. Kashyap SR, Karen MD, John TB, Hemant JP, Nitin $\mathrm{HC}$, Girdhar MT etal. Demonstration of Components of Antigen 85 Complex in Cerebrospinal Fluid of Tuberculous Meningitis Patients. Clin Diagn Lab Immunol 2005; 12:752-758.

21. Johnson S, Brusasca P, Lyashchenko K, Wiker HG, Bifani P, Shashkina E et al. Characterization of the secreted MPT53 antigen of Mycobacterium tuberculosis. Infect Immun 2001; 69:5936-5939.

22. Upadhye VJ, Gomashe AV, Kumar S, Harinath BC. Isolation, characterisation and kinetic studies on SEVA TB ES-31 antigen, a metallo-serine protease of interest in serodiagnosis. Indian J Tuberc 2009; 56:22-29. 